\title{
The Cues of COVID-19: Negative Emotions and Empathy Guide Prosocial Behavior
}

\author{
Dongfang $\mathrm{Li}^{1, *}$ \\ ${ }^{1}$ School of Teacher Education, Anqing Normal University of Applied Psychology, Anqing, Anhui 246052, China \\ ${ }^{*}$ Corresponding author. Email: DongfangLi20210802@163.com
}

\begin{abstract}
The COVID-19 research literature reveals that during the COVID-19 pandemic, people faced with unemployment risks, community blockades, and various negative information on the Internet, people will have fear, disgust, sadness, and other emotions in the short term, and will lead to anxiety and depression in a long time symptom, and cues about the COVID-19 can also cause emotional changes in people. Research on pro-social behaviors suggests that the influence of negative emotions on pro-social behavior tendencies is complex, and empathy will increase pro-social behavior. Study 1 is a measurement of people's pro-social behavior tendency under two different conditions. Compared with the controlled subjects, the subjects assigned to watch the cues of COVID-19 in Chinese reported more pro-social behavioral tendencies. In Study 2, we find that cues related to the epidemic did affect people's emotional changes, and the sum of these emotions also has a significant impact on pro-social behavioral tendencies. To understand which emotional mediator has the greatest influence on pro-social behavioral tendencies, study 2 also finds the complete mediating effect of empathy. Our results indicate that the cues of COVID-19 increased people's negative emotions and empathy, and empathy led to an increase in pro-social behavior, this reaction even crosses race and country. The result also has important implications for initiating people's pro-social behavior in major crisis events in the future.
\end{abstract}

Keywords: COVID-19, pro-social behavior, emotion, empathy

\section{INTRODUCTION}

COVID-19, or Coronavirus Disease 2019, was declared an epidemic by the World Health Organization on March 11, 2020. The illness had also caused widespread panic in the area of the world where this pronouncement was made. Schools, restaurants, and offices have closed, and governments have advised citizens to stay in place, usually requesting that inhabitants leave their homes with identification[1]. Communities and even cities and countries are sealed off. COVID-19 has led to the economic crisis, social closure, increased unemployment risks, and drastic changes in people's lifestyles (remote work and study, fewer gatherings, remaining at home for an extended period, etc.) The prospect of a fresh epidemic of crown pneumonia, with its limits on individual freedoms, group communication, and the closing of businesses and stores, has sparked psychological resistance among the general population, but COVID-19 also makes us more concerned about the public interest[2-7]. The number of COVID-19 illnesses in India reached 360,960 on April
26,2021 , the largest daily record in the world's history, bringing the total number of pandemics to 16 million, second only to the United States, with more than 200,000 fatalities. The deadly second wave of the pandemic struck one year after the state passed one of the toughest lockdown restrictions in the world - barely three months after the Health department proclaimed infection and mortality to be at their lowest point in history[8]. Therefore, the COVID-19 situation in India is still very severe.

Folk's physical and mental health has been severely jeopardized as a result of the spread of COVID-19, and the number of depression and anxiety has increased significantly. Therefore, we believe that clues related to the epidemic can cause people's negative emotions to appear. And the negative emotions caused by COVID19 seem to arouse people's empathy. Sheldon and Rogers observed that viewing the "bloody scene" of predators shooting and handling whales induced empathy. Compared with the low disgust situation where bloody scenes are omitted, compassion leads to a 
strong intention to help save the whales and support Greenpeace[9]. The situation of COVID-19 can be analogized. We have reason to believe that when people see patients suffering from the disease and their helpless family members, the sadness, disgust, and other emotions they produce will also lead to increased empathy. Compared with the low-distortion situation where death scenes are omitted, compassion results in a great desire to assist and support patients. The existing literature, overwhelmingly suggests that emotions affect people's pro-social behavior. Different emotions have different effects on pro-social behavior. It can be unanimously agreed that positive emotions have a favorable impact on pro-social behavior tendencies[10, 11]; while the influence of negative emotions on prosocial behavior is uncertain. The same negative emotions produced in different situations have different effects. In some situations, "fear" can increase people's pro-social behavior[12], and in some cases, "fear" can reduce people's pro-social behavior[13]. Studies have shown that when these negative emotions can cause people to report more empathy, they will lead to more pro-social behaviors $[14,15]$. Empathy itself can also increase people's pro-social behavior[16]. The impact of prosocial behavior is cross-cultural[17]. In the research related to COVID-19, we find that people's anxiety and depression status increase significantly during the prevalence of COVID-19[18-22]. There are two main reasons for this situation, one is the government's policy of closing cities and homes and causing them to feel more stressed in life (new study or working style, risk of unemployment, risk of lower wages, risk of infection, etc.) $[18-20,22,23]$, and the other is the large amount of (false) negative information about the virus that the people are exposed to[18, 19, 24-27]. The above two reasons have caused people's inner panic and anxiety. Under the influence of such emotions, people publish more negative emotion-related topics and posts on social media[24], which forms a vicious circle and also makes the government control Met some obstacles. Studies have shown that allowing people to read information about infectious diseases can arouse people's feelings of disgust and fear[7]. Watching photos of "little girls with bruises" synthesized by photoshop can arouse people's negative emotions and trigger empathy[28]. Therefore, we believe that COVID-19-related clues can also cause emotional changes in people.

In this study, we investigate how various emotional reactions to COVID-19 signals influence pro-social behavior propensity. We use research on assessment tendencies and emotions to assert that the behavioral response to the COVID-19 cues will increase pro-social behavior tendencies, and which emotions will have a greater impact on pro-social behavior tendencies, and which aspects of pro-social behavior tendencies are specifically affected. We evaluate this hypothesis in two investigations, each with two experiments. In these experiments, we discovered that COVID-19 signals can evoke sadness, fear, anger, tension, and empathy in people. But we find that only empathy has a significant influence on pro-social behavior tendency. Through bootstrap, we find that empathy has a completely mediating effect.

Our findings emphasize the need of comprehending why, which aspects of each different emotion affect prosocial behavioral tendencies, how cues of major crisis affect pro-social behavior tendency, and what the impact of major crisis events on pro-social behavior is. We demonstrate the effectiveness of the COVID-19 cues on pro-social behavior, and we gain empirical proof into the function of different emotions in affecting pro-social behavior, finally, we discuss the practical significance of guiding people to increase pro-social behaviors for the government.

\subsection{Literature review:}

\subsubsection{The impact of COVID-19:}

Current research shows that during the COVID-19, people's long-term negative emotions such as anxiety, depression, etc. will be significantly affected [18-22]. Seven important factors associate with increased levels of anxiety include women, residents of urban areas, and emotion-centric coping styles. Nine factors associate with increase depression levels, such as smartphone addiction (or 1.411, 95\% CI1.099-1.180), Internet addiction (or 1.844, 95\% CI1.209-2.811), and living in Hubei Province (Or 3.107, 95\% CI 1.252-7.708)[18]. There is also a study that indicates that even clues related to the COVID-19 also can affect people's emotions. When people encounter infectious disease prompts, In addition to the primary indicators of pollutant presence, there are frequently ambiguities and a lack of personal control.Namely: if a train passenger coughs, another rider may be irritated and concerned about being ill[7].

China is the first nation to be hit by the COVID-19 in 2019. This disaster not only has a great impact on the national economy but also caused a public mental health crisis. Studies have shown that COVID-19 is more easily transmitted than SARS, and the death rate $(2.3$ percent) is significantly greater than that of seasonal flu. The ambiguity of the virus's incubation time and possibly asymptomatic transmission not only increases the danger of infection but also creates severe psychological stress. Second, the administration's early underestimation of the severity of COVID-19 eroded public faith in the government's openness and decisionmaking capacity. Third, all-time extensive isolation tactics in big cities have effectively kept inhabitants at home, potentially harming residents' social psychology. Finally, a unique kind of "information about COVID- 
19 "-too much information that is not necessarily correct is spread on social media and elsewhere-poses a major risk to public mental health during this health crisis[8, 29, 30]. Therefore, the COVID-19 in China in early 2020 has deeply affected the hearts of every Chinese.

\subsubsection{Pro-social behavior}

Regarding the definition of pro-social behavior, the classic definition is proposed by Paul Mussen in 1978. He believes that pro-social behavior refers to any behavior that tries to help other individuals or a group to benefit them, including helping others, generosity, sacrifice, and defense being fearless, loyal, respecting the rights and feelings of others, and having a sense of responsibility[31]. In 1982, Orlick McNally and O'Hara defined pro-social behavior as any behavior that shares with others helps others, and intimately touches the bodies of others in their research[32]. Krebs conceive pro-social behavior as a continuum of behaviors, one end in the direction of maximizing self-interest, and the other end in the direction of maximizing the interests of others. The amount of altruistic components of behavior is determined by two criteria: the direction of behavior and the total amount of benefits contained in the behavior. Altruistic behavior is to maximize the interests of others on this continuum[33]. To sum up, pro-social behavior refers to all altruistic behaviors that people show in their daily behavior, including both selfinterested and purely altruistic, and includes both nonaggressive behaviors and pro-social behaviors. Violations. Pro-social behaviors mainly include sharing, cooperating, helping others, and comforting behaviors.

\subsubsection{Negative emotion}

During the COVID-19 outbreak, people's physical and mental health has been greatly threatened, and the number of depression and anxiety has increased significantly. Current research shows that during the COVID-19, people's long-term negative emotions such as anxiety, depression, etc. will be significantly affected [18-22]. During the dissemination of COVID-19 in China, a questionnaire survey of 359 children and 3254 adolescents was undertaken. The results show that children and teenagers had anxiety levels of $(23.87 \pm 15.79) \quad$ and $(29.27 \pm 19.79)$, respectively. Depression symptoms are experienced by 22.28 percent of those polled[18]. There are also studies showing that clues related to infectious diseases can cause people's disgust and fear[7]. For the same reason, we believe that clues related to the epidemic can cause people's negative emotions to appear.

Several researchers here found that there is a connection between emotions and pro-social behaviors, indicating that pleasant emotions are conducive to the occurrence of pro-social behaviors[10, 11]. In Susan
Michie's research, they find that leaders increase their pro-social behavior when they feel pride and gratitude[10]. The research of Lara B. Aknin et al. Considers the importance of good feelings. Pleasant emotional states can remind actors to pay attention to positive experiences and promote similar behaviors in the future, according to the functional definition of positive emotions. They review the evidence that pleasant emotions encourage and reward pro-social conduct throughout the developmental process. The relationship between negative moods and helping behaviors is much more complicated. Some studies believe that negative emotions (sadness, guilt, shame) will increase helping behaviors to seek compensation, and reporting negative emotions will report more empathy, which will also increase pro-social behaviors[14, 15]. Research by Richard P. Bagozzi et al. finds that people's negative emotions such as anger, sadness, fear, and tension after watching public service advertisements about child abuse have a positive impact on their willingness to provide more help[14]. The research of Robert. B Cialdini et al. find that emotion and helping others seems to have a "U"-shaped relationship, that is, high negative emotions or high positive emotions will increase people's helping behaviors[15]. There are also studies showing that negative emotions have no obvious effect on pro-social behavior[28, 34]. Studies by Tsamchoe Dolma and Jason J. Barr and others have shown that even if people's negative emotions and empathy are aroused, people's prosocial behavior tendencies do not necessarily have significant differences[28, 34]. But some studies have shown that negative emotions are negatively related to pro-social behaviors[13, 35-37]. The study by Scott F. Stoltenberg and others finds that the triallelic 5-HTTLPR genotype is related to prosocial behaviors, and its influence is mediated in part by social anxiety. As a result, persons with the $\mathrm{S}$ allele have greater levels of social avoidance and lower rates of assisting others[13]. The study by Joanneke Weerdmeester and others confirms that People with low social anxiety respond to denial in a compensating, prosocial manner, whereas those with high social anxiety do not[35]. If risk factors are included in the helping situation, people will have fear, anxiety, worry, and other emotions, which will stimulate self-protection motivation and reduce pro-social behavior[36]. Similarly, negative emotions can easily induce aggressive behaviors, leading to a decrease in pro-social behavior[37]. However, due to the self-praise of prosocial behaviors, negative emotions are encouraged to make pro-social behaviors[37]. It can be seen that the influence of emotion on pro-social behavior is very complicated. The breakthrough of this research is to explore whether the emotions generated under major crises will increase people's pro-social behavior. 


\subsubsection{Empathy}

Research by Richard P. Bagozzi points out that the empathy generated by negative emotions increases people's willingness to help others. Figure 1 depicts the independent factors and assumptions of Richard P. Bagozzi[14]. Some study suggests that empathy has a role in help decision-making. Empathy can encourage prosocial behavior, according to a study conducted by Batson and Dovidio [38, 39]. Kirk, Batson, and McDavis discovered that empathy for others in need elicits empathy, prompting the perceiver to help[40]. Sheldon and Rogers discover that the disgust caused by seeing the "bloody scene" of predators shooting and dealing whales led to empathy. Compared with the low disgust situation where bloody scenes are omitted, Compassion inspires a great desire to assist in the conservation of whales and to support Greenpeace[9].

But, exactly, what is empathy? Titchener coined the word "empathy," saying, "I can not only observe dignity, humility, pride, civility, and decency but also feel them in my head." This, I believe, is a basic example. Einfiihlung means "feeling" in German[41]. Hoffman pointed out that in the current environment, empathy is typically described as the recognition of the hearts of others. Put yourself in the shoes of another individual and go through Human emotions [42]. Some psychologists have a more limited understanding of empathy. For example, Lazarus wrote: "I don't think empathy is an emotion. Instead, it should be seen as an emotional capacity and process." Feel the emotions of others in the emotional environment." [43]. Similarly, Wispe sees empathy as "a self-aware self, trying to understand the positive and negative experiences of another self without judgment." He defines the related concept of compassion as "helping others reduce pain." Increased awareness" [44]. Therefore, this article takes empathy into the emotional factor as an intermediary consideration.

\subsubsection{PTM}

Carlo's research aims to investigate the psychometric characteristics of multidimensional measures of prosocial behaviors used in early and mid-term adolescents[45]. Professor Kou Yu of Beijing Normal University uses adolescents as a research sample to revise the Pro-social Tendency Scale (PTM) compiled by Carlo[46]. According to the cultural background of our country and the specific conditions of youth development, a theoretical concept has been established, and four tests have been carried out. According to the first measurement results, three items have been added to the compliance dimension, the reverse question has been added, and the description of all items Make the necessary changes. According to the subsequent test results, two items in the altruistic dimension are deleted, and the revised six subscales of the PTM-open, anonymous, altruistic, compliant, emotional, and urgent internal consistency letter The degrees are $0.708,0.778$, $0.755,0.738,0.726,0.561$, and the correlations between the subscales and the total scale are $0.494,0.758,0.801$, $0.800,0.789,0.803$, respectively. The revised results of the scale are closer to Carlo's revised results in the United States and have good psychological measurement indicators[46].

\subsection{Hypothesis}

In major crisis events, our general habit is that the public's pro-social behavior will increase, but there a few studies discuss the relationships between the major crisis events and pro-social behavior. There have indeed been researched breakthroughs. During the COVID-19, people's relatives' social behaviors will increase[47]. Some relevant clues will also change people's pro-social behaviors, such as the role of role models[48], public service announcements about child abuse[14], these all can increase people's pro-social behavior. Therefore, we suppose that the cues of COVID-19 also can increase people's pro-social behaviors. More formally:

H1: Cues of COVID-19 disease increase pro-social behavior tendencies.

Current research shows that during the COVID-19, people's long-term emotions such as anxiety and depression will be significantly affected[18-22]. Studies have also shown that only providing clues about the COVID-19 can cause people to have negative emotions such as fear and disgust[7]. The image of a young girl has bruises can lead to negative emotions(sadness, fear, anger, and tension) and empathy[28]. It is thus possible that receiving signs concerning infectious disease might trigger negative emotions as well as empathy in people. More formally:

H2a: When confronting the COVID-19 cues, people feel sadness, fear, anger, tension, and empathy from assessing a specific danger source.

We have thus far proposed that COVID-19-related clues will lead to an increase in pro-social behavior, and it will also induce people's negative emotions and empathy. The research also shows that public service announcements about child abuse not only can lead to negative emotions and empathy but also increase people's pro-social behavior[14]. Studies have agreed that positive moods can promote helping behaviors, but the relationship between negative moods and helping behaviors is much more complicated. Some studies believe that negative emotions (sadness, guilt, shame) increase helping behaviors to seek compensation. Reporting negative emotions will report more empathy[14, 15, 28], and empathy will promote an increase in pro-social behaviors[16]. There are also some studies showing that negative emotions are 
negatively related to pro-social behaviors[13, 35,49$]$. If risk factors are included in the helping situation, people will have fear, anxiety, worry, and other emotions, which will stimulate self-protection motivation and reduce pro-social behavior[36]. As for empathy, the current research on empathy and pro-social behavior has found that even if an increase in empathy does not increase pro-social behavior, it will not decrease prosocial behavior[14, 15, 28, 34]. It can be seen that the influence of mood on pro-social behavior is very complicated. Therefore, both negative emotion and empathy may increase people's pro-social behavior tendency, and the negative emotion and empathy do not conflict, but the increase of empathy to pro-social behavior tendency may be more prominent. More formally:

$\mathrm{H} 2 \mathrm{~b}$ : Relative to sadness, fear, anger, tension, experiencing empathy in response to the COVID-19 cues increase more pro-social behavior tendencies.

The earlier a counterexample appears, the more efficient our framework performs.

\section{STUDIES}

\subsection{Study 1}

We designed Study 1 to test whether cues of COVID-19 increase the pro-social behavior (hypothesis 1) and to evaluate how COVID-19 clues affect the six subscales(open, anonymous, altruistic, compliant, emotional, urgent) of pro-social tendencies. Furthermore, as an additional control condition, we employ neutral stimuli to rule out the possibility that the empirically discovered result patterns represent people's responses to generic objects rather than COVID-19.

\section{Method}

Design. Take 388 samples aged 15-50, and other demographic factors are basically average, which will not cause much interference. Using the online experiment method, the subjects are divided into two groups: an experimental group and a control group.

Manipulate. After agreeing to participate, all participants read a brief news story and view some photographs. Participants in the control condition read about a new pressure cooker. Participants in the COVID-19 cue condition read about the spread of the COVID-19 in China from March to May 2020. All news stories and images are taken from actual information on the product or the COVID-19 disease, and the length of the material and the number of images are the same.

Measuring pro-social behavior tendency. After reading the news report and pictures, all participants respond to their pro-social behavior tendency immediately. We using the Pro-Social Behavior Tendency Scale $(\mathrm{PTM})(\alpha$ 's $=0.955)$ to measure their pro-social behavior tendency (be divided into six dimensions). The PTM scale contains 26 questions and where they are indicated on a scale from $1=$ "not at all" to 5 = "very much". The six subscales are open, anonymous, altruistic, compliant, emotional, urgent.

\section{Results}

Exclusion. Of the 388 participants, 49 participants are excluded via the filtering criteria. Analyses are run with the remaining 339 participants $($ Mage $=22.3$, $54.1 \%=$ females $)$.

pro-social behavior tendency. Among 339 participants, $52.9 \%$ of the participants watch the clues of the severe moment of the COVID-19 in China, and $47.1 \%$ of the participants watch the relevant information of the water heater. As expected, participants who read about the cues of contagious disease $(\mathrm{M}=96.91, \mathrm{SD}=$ 16.98) felt more proactive than participants who read information about water heater conditions $(\mathrm{M}=91.44$, $\mathrm{SD}=16.94, \mathrm{t}=2.96, \mathrm{p}=0.03<0.05$ )in pro-social behavior tendencies, supporting hypothesis 1 .

Judging from the six sub-scales, participants reading about the cues of contagious disease experience significantly more compliant $(\mathrm{t}=2.015, \mathrm{p}=0.045<$ $0.05)$, public $(\mathrm{t}=3.371, \mathrm{p}=0.001<0.05)$, dire $(\mathrm{t}=$ $3.060, \mathrm{p}=0.002<0.05)$, emotional $(\mathrm{t}=2.502, \mathrm{p}=$ $0.013<0.05)$, altruism $(\mathrm{t}=3.012, \mathrm{p}=0.003<$ 0.05 ) than participants reading about information of water heaters, But there is no significant difference between the two groups in terms of anonymous $(t=$ $1.698, \mathrm{p}=0.09>0.05)$.

\subsection{Study 2}

We designed Study 2 to test whether cues of COVID-19 elicit negative emotions ( sadness, fear, anger, tension) and empathy response (hypothesis 2a) and whether empathy in response to the COVID-19 cues increases more pro-social behavior tendencies than sadness, fear, anger, and tension (hypothesis 2b). Furthermore, As an auxiliary control condition, we employ a neutral stimulus to rule out the possibility that the empirical research' pattern of findings shows people's responses to generic things rather than COVID19 specifically.

\section{Method}

Design. Take 388 samples aged 15-50, and other demographic factors are basically average, which will not cause much interference. Using the online experiment method, the subjects are divided into two groups: an experimental group and a control group.

Manipulating emotions. After consenting to take part, everyone reads a brief news item and looks at some photos. In the control condition, participants read information and view images of new pressure cookers. 
Participants in the COVID-19 cue condition learned about the COVID-19's recent expansion in India (May 2021). All news reports and photos are based on genuine facts on the product or the COVID-19 illness, and the length and quantity of images are the same.

Measuring emotions. Following the reading of the news story and images, all participants complete an emotions index based on past research, in which they indicate the extent to which they felt each of the four negative emotions words and empathy during the news report task ("sadness;" 's = 0.899); "fear;" 's = 0.907); “anger;" 's = 0.915); "tension;" 's = 0.921)[42].

Measuring pro-social behavior tendency. After reading the news report and pictures, all participants respond to their pro-social behavior tendency immediately. We using the Pro-Social Behavior Tendency Scale (PTM) $(\alpha$ 's $=0.955)$ to measure their pro-social behavior tendency (be divided into six dimensions). The PTM scale contains 26 questions and where they are indicated on a scale from $1=$ "not at all" to 5 = "very much". The six subscales are open, anonymous, altruistic, compliant, emotional, urgent.

\section{Results}

Exclusion. Of the 388 participants, 49 participants are excluded via the filtering criteria. Analyses are run with the remaining 339 participants (Mage $=22.3$, $54.1 \%=$ females).

Emotions. Through the T-test, we find that participants reading about the cues of contagious disease experience significantly more $\mathrm{sad}(\mathrm{t}=19.516, \mathrm{p}$ $=0.000<0.05)$, fearful $(\mathrm{t}=12.226, \mathrm{p}=0.000<.05)$, angry $(\mathrm{t}=10.926, \mathrm{p}=0.000<0.05)$, tensional $(\mathrm{t}=8.360$, $\mathrm{p}=0.000<0.05)$, empathetic $(\mathrm{t}=19.674, \mathrm{p}=0.000<$ 0.05 ) than participants reading about information of pressure cookers, supporting hypothesis $2 \mathrm{a}$.

Elicit pro-social behavior tendency. Using regression analysis find that only "empathetic" has a significant impact on pro-social behavioral tendencies $(\mathrm{t}$ $=2.409, \mathrm{p}=0.017<0.05$ ).Finally, use bootstrap to find that only "empathetic" has a mediating effect (BootLLCI $=-13.502$, BootULCI $=-2.409$ ), supporting hypothesis $2 b$.

\section{CONCLUSION}

\subsection{General discussion}

Across two experiments, we find that cues of COVID-19 have an increasing impact on pro-social behavior tendency. The results show significant differences in five dimensions (compliant, public, dire, emotional, altruism) of pro-social behavior tendency, but there is no significant difference between the two groups in terms of anonymous. Our results also support that cues of COVID-19 leading to sadness, fear, anger, tension, empathy response in people. Among them, empathy increases people's pro-social behavior tendency.

Our findings help us understand how people's prosocial behavior tendencies are influenced by their emotional states. Most previous studies on pro-social behavior did not start with major crisis events. Likewise, most previous studies on emotions caused by COVID19 have studied its effects on people's long-term emotions, such as anxiety and depression over a period of time[18-22]. However, little study has been conducted to investigate the behavior propensity of people in settings in which they are exposed to important cues concerning COVID-19 and evoke various emotions. Although the influence of these emotions on pro-social conduct may be common concepts, our literature here shows that these emotions can be awakened by major crisis events and have a positive impact on pro-social behavior. Cues of COVID-19-by eliciting both negative emotions and empathy - and empathy results in an increased impact on pro-social behavior tendency.

Our results have important implications for the government. When a major crisis event sweeps a city or country, it is extremely important to elicit people's prosocial behavior. It can make the people more subject to the unified management and control of the government, and it can also encourage people to spontaneously organize public welfare activities and support. The prosocial behavior of the people plays an important role in solving major crises.

\subsection{Limitation and Future Research}

This research only studies the emotions generate under neutral stimuli and major crisis events and their changes to pro-social behavior. It does not measure the difference between negative emotions elicit under major crisis events and the negative emotions elicit under other events (thrillers, violent games, etc.). Current research shows that in a different situation, the influence of emotions on pro-social behavior is different $[12,13$, $35,49-51]$.

\section{REFERENCES}

[1] Fernandes, N., Economic effects of coronavirus outbreak (COVID-19) on the world economy. Available at SSRN 3557504, 2020.

[2] Addo, P.C., et al., COVID-19: fear appeal favoring purchase behavior towards personal protective equipment. The Service Industries Journal, 2020. 40(7-8): p. 471-490.

[3] Guthrie, C., S. Fosso-Wamba, and J.B. Arnaud, Online consumer resilience during a pandemic: An exploratory study of e-commerce behavior before, 
during and after a COVID-19 lockdown. Journal of Retailing and Consumer Services, 2021. 61: p. 102570 .

[4] Krishnamurthy, S., The future of business education: A commentary in the shadow of the Covid-19 pandemic. Journal of Business Research, 2020. 117: p. 1-5.

[5] Wang, C., et al., A Multinational Study on the Protective and Risk Factors Associated with Mental Health Parameters in the General Population During the COVID-19 Pandemic: A Study in the Asia-Europe-North America Cohort. 2020.

[6] Szymkowiak, A., et al., The impact of emotions on shopping behavior during epidemic. What a business can do to protect customers. Journal of Consumer Behaviour, 2021. 20(1): p. 48-60.

[7] Galoni, C., et al., Disgusted and Afraid: Consumer Choices under the Threat of Contagious Disease. Journal of Consumer Research, 2020. 47(3): p. 373-392.

[8] Thiagarajan, K., Why is India having a covid-19 surge? 2021, British Medical Journal Publishing Group.

[9] Shelton, M.L. and R.W. Rogers, Fear-arousing and empathy-arousing appeals to help: The pathos of persuasion. Journal of applied social psychology, 1981. 11(4): p. 366-378.

[10] Michie, S., Pride and gratitude: How positive emotions influence the prosocial behaviors of organizational leaders. Journal of Leadership \& Organizational Studies, 2009. 15(4): p. 393-403.

[11] Aknin, L.B., J.W. Van de Vondervoort, and J.K. Hamlin, Positive feelings reward and promote prosocial behavior. Current opinion in psychology, 2018. 20: p. 55-59.

[12] Van Doorn, J., M. Zeelenberg, and S.M. Breugelmans, Anger and Prosocial Behavior. Emotion Review, 2014. 6(3): p. 261-268.

[13]Stoltenberg, S.F., C.C. Christ, and G. Carlo, Afraid to help: social anxiety partially mediates the association between 5-HTTLPR triallelic genotype and prosocial behavior. Social neuroscience, 2013. 8(5): p. 400-406.

[14]Bagozzi, R.P. and D.J. Moore, Public service advertisements: Emotions and empathy guide prosocial behavior. Journal of marketing, 1994. 58(1): p. 56-70.

[15] Cialdini, R.B., B.L. Darby, and J.E. Vincent, Transgression and altruism: A case for hedonism.
Journal of Experimental Social Psychology, 1973. 9(6): p. 502-516.

[16] Staub, E., The use of role playing and induction in children's learning of helping and sharing behavior. Child Development, 1971: p. 805-816.

[17]Trommsdorff, G., W. Friedlmeier, and B. Mayer, Sympathy, distress, and prosocial behavior of preschool children in four cultures. International Journal of Behavioral Development, 2007. 31(3): p. 284-293.

[18] Duan, L., et al., An investigation of mental health status of children and adolescents in china during the outbreak of COVID-19. J Affect Disord, 2020. 275: p. 112-118.

[19] Elhai, J.D., et al., COVID-19 anxiety symptoms associated with problematic smartphone use severity in Chinese adults. J Affect Disord, 2020. 274: p. 576-582.

[20] Tang, W., et al., Prevalence and correlates of PTSD and depressive symptoms one month after the outbreak of the COVID-19 epidemic in a sample of home-quarantined Chinese university students. J Affect Disord, 2020. 274: p. 1-7.

[21] Wang, Z.H., et al., Prevalence of anxiety and depression symptom, and the demands for psychological knowledge and interventions in college students during COVID-19 epidemic: A large cross-sectional study. J Affect Disord, 2020. 275: p. 188-193.

[22] Xiong, J., et al., Impact of COVID-19 pandemic on mental health in the general population: A systematic review. J Affect Disord, 2020. 277: p. 55-64.

[23] Rudenstine, S., et al., Depression and Anxiety During the COVID-19 Pandemic in an Urban, Low-Income Public University Sample. J Trauma Stress, 2021. 34(1): p. 12-22.

[24] Singh, P., et al., Psychological fear and anxiety caused by COVID-19: Insights from Twitter analytics. Asian J Psychiatr, 2020. 54: p. 102280.

[25] Chang, J., Y. Yuan, and D. Wang, Mental health status and its influencing factors among college students during the epidemic of COVID-19. Nan fang yi ke da xue xue bao= Journal of Southern Medical University, 2020. 40(2): p. 171-176.

[26] Liu, Y., et al., Public awareness and anxiety during COVID-19 epidemic in China: A cross-sectional study. Comprehensive Psychiatry, 2021. 107: p. 152235 . 
[27] Tessler, H., M. Choi, and G. Kao, The anxiety of being Asian American: Hate crimes and negative biases during the COVID-19 pandemic. American Journal of Criminal Justice, 2020. 45(4): p. 636646.

[28] Dolma, T., Different levels of negative emotions and their impact on prosocial behavior. 2015.

[29] Cao, W., et al., The psychological impact of the COVID-19 epidemic on college students in China. Psychiatry research, 2020. 287: p. 112934.

[30] Dong, L. and J. Bouey, Public mental health crisis during COVID-19 pandemic, China. Emerging infectious diseases, 2020. 26(7): p. 1616.

[31] Mainardi, D., Roots of caring, sharing, and helping. The development of prosocial behavior in children: Paul Mussen and Nancy Eisenberg-Berg. Freeman and Co., San Francisco, Calif., 1977, vii+ 212 pp., US \$5.50 (soft cover), ISBN 0-71670044-1. 1978, Elsevier.

[32] Bay-Hinitz, A.K., R.F. Peterson, and H.R. Quilitch, Cooperative games: A way to modify aggressive and cooperative behaviors in young children. Journal of applied behavior analysis, 1994. 27(3): p. 435 .

[33] Krebs, D., Prosocial behavior, equity, and justice, in Equity and justice in social behavior. 1982, Elsevier. p. 261-308.

[34] Barr, J.J. and A. Higgins-D'Alessandro, Adolescent empathy and prosocial behavior in the multidimensional context of school culture. J Genet Psychol, 2007. 168(3): p. 231-50.

[35] Weerdmeester, J. and W.G. Lange, Social Anxiety and Pro-social Behavior Following Varying Degrees of Rejection: Piloting a New Experimental Paradigm. Front Psychol, 2019. 10: p. 1325.

[36] CHAN, J.H. and N. WHITE, The IC. ARE Experience: Promoting School Safety and Prosocial Behaviour. Education Canada, 2010. 2: p. 19-21.

[37] Liu Zewen and Zhao Ailing, A Review of the Influencing Factors of Children's Prosocial Behaviors. Chinese Out-of-School Education: Late Ten Days, 2009(S2): p. 342-342.

[38] Batson, C.D., Prosocial motivation: Is it ever truly altruistic? Advances in experimental social psychology, 1987. 20: p. 65-122.

[39] Dovidio, J.F., Helping behavior and altruism: An empirical and conceptual overview. Advances in experimental social psychology, 1984. 17: p. 361427.
[40] Coke, J.S., C.D. Batson, and K. McDavis, Empathic mediation of helping: a two-stage model. Journal of personality and social psychology, 1978. 36(7): p. 752 .

[41] Titchener, E.B., Lectures on the experimental psychology of the thought-processes. 1909: Macmillan.

[42] Hoffman, M.L., Interaction of affect and cognition in empathy. Emotions, cognition, and behavior, 1984: p. 103-131.

[43] Lazarus, R.S. and R.S. Lazarus, Emotion and adaptation. 1991: Oxford University Press on Demand.

[44] Wispe, L., The distinction between sympathy and empathy: To call forth a concept, a word is needed. Journal of personality and social psychology, 1986. 50(2): p. 314

[45] Carlo, G., et al., Sociocognitive and Behavioral Correlates of a Measure of Prosocial Tendencies for Adolescents. The Journal of Early Adolescence, 2016. 23(1): p. 107-134.

[46] Kou Yu, et al., Revision of the Prosocial Tendency Scale for Adolescents. Psychological Development and Education, 2007. 23(1): p. 112-117.

[47] Van de Groep, S., et al., A daily diary study on adolescents' mood, empathy, and prosocial behavior during the COVID-19 pandemic. PloS one, 2020. 15(10): p. e0240349.

[48] Abel, M. and W. Brown, Prosocial behavior in the time of COVID-19: The effect of private and public role models. 2020 .

[49] Eisenberg, N., et al., The role of sympathy and altruistic personality traits in helping: A reexamination. Journal of personality, 1989. 57(1): p. 41-67.

[50] Dinić, B.M. and B. Bodroža, COVID-19 Protective Behaviors Are Forms of Prosocial and Unselfish Behaviors. Frontiers in Psychology, 2021. 12.

[51] Van de Ven, N., M. Zeelenberg, and R. Pieters, Warding off the evil eye: When the fear of being envied increases prosocial behavior. Psychological Science, 2010. 21(11): p. 1671-1677. 